\title{
Incorporating teleoncology practices in the undergraduate medical curriculum
}

\author{
Anna Maria Wozniak*,1,2 (iD) \& Kathrine Sofia Rallis ${ }^{1,2}$ (D) \\ ${ }^{1}$ Barts Cancer Institute, Queen Mary University of London, London, EC1M 6BE, UK \\ ${ }^{2}$ Barts \& The London School of Medicine \& Dentistry, Queen Mary University of London, London, E1 2AD, UK \\ *Author for correspondence: a.m.wozniak@smd16.qmul.ac.uk
}

First draft submitted: 6 August 2020; Accepted for publication: 21 August 2020; Published online: 1 Novemeber 2020

Keywords: cancer teaching • coronavirus • COVID-19 • medical education and training • medical school • medical students • telehealth $\bullet$ telemedicine $\bullet$ teleoncology $\bullet$ undergraduate medical education

\section{To the Editor,}

We have read with interest the article by Elkaddoum et al., 'Telemedicine for cancer patients during COVID19 pandemic: between threats and opportunities', describing the shift from face-to-face oncology practice to teleconsultations [1]. As UK medical students experiencing the disruptions caused by COVID-19, we appreciate the advantages of teleoncology. However, we fear the transition to teleoncology may further reduce our exposure to cancer specialties. Thus, we would like to take this opportunity to highlight our concerns, discuss strategies and limitations of integrating medical students in teleoncology services and offer solutions to overcome these challenges.

The first cases of COVID-19 were identified in December 2019 in the Chinese town of Wuhan, which later became the first epicenter of a global outbreak [2]. The rapidly spreading virus proved to be particularly dangerous, especially to older individuals as well as those with underlying conditions such as cancer, making these individuals more susceptible to severe complications of the infection [3,4]. Health care underwent an unprecedented change to minimize the risk of viral infection and spread. Teleoncology, the blueprints of which were already in development [5], proved to be an invaluable resource during this time. Initially aimed at diminishing disparities in access to quality cancer care between high-income urban areas and rural, remote districts or low- and middle-income countries [5], teleoncology quickly became the principal means of communication between immunosuppressed cancer patients and oncologists [6]. Indeed, the potential of teleoncology became apparent.

Meanwhile, undergraduate clinical teaching was largely suspended across most countries as universities were forced to close in light of the pandemic [7]. Although the education of all medical specialties was affected, we believe oncology to be disproportionately impacted. Oncology is already an underrepresented specialty in undergraduate medical curricula, with students worldwide reporting limited exposure to oncology specialties, teaching dissatisfaction and low confidence in oncology care, particularly relating to breaking bad news [8]. Student conferences, summer electives and research internships - all of which have faced cancellations amid COVID19 - significantly influence students' career interest, especially toward disciplines that feature little dedicated teaching in the UK undergraduate medical curriculum [9]. We are concerned that decreases in oncology exposure will reduce student interest in pursuing oncology as a career because early specialty exposure is associated with specialty interest and career selection [10]. Moreover, the consequences are graver given that clinical placements have irrevocably changed. Even after COVID-19 has subsided, restrictions will likely persist longer in specialties involving immunosuppressed patients such as oncology. Reduced oncology exposure during medical school may diminish student interest in the specialty, resulting in fewer applicant trainees and future specialty attrition. Significant long-term deficiencies may be observed in the cancer workforce, which is already overstrained [11]. Low-quality cancer services and increased physical and mental burden on oncologists may result, contributing to higher rates of physician burnout [11]. Therefore, early integration of medical students in teleoncology is crucial.

The changing face of oncology practices described by Elkaddoum et al. necessitates an equal change in undergraduate medical training [1]. Telemedicine offers unique advantages to traditional teaching. Virtual clinics 
could increase students' access to oncology, enabling them to practice interviewing and clerking cancer patients while maintaining social distancing measures, reducing the risk of viral contraction and spread, and minimizing personal protective equipment use [1]. Inviting students to attend online multidisciplinary team (MDT) meetings and teleconferences would enhance student's understanding and cultivate specialty interest. Indeed, virtual seminars are effective at facilitating international collaborations and bolstering research during COVID-19 lockdown, and there is an ongoing discussion of replacing in-person conferences with teleconferences in the future [12]. Lastly, creating peripheral cancer care centers, as described by the authors [1], would address student capacity issues currently faced at specialist cancer centers that limit students to brief oncology experiences [13].

Yet teleoncology also has shortcomings. We agree with the authors' concerns regarding the accessibility of technology for both patients and students [1]. Lack of access to personal electronic devices and stable broadband connection may widen social inequalities. Furthermore, although the integration of telemedicine in undergraduate medical teaching would be a valuable substitute for student-patient contact throughout the pandemic, it does not equate to traditional face-to-face clinical placements. Inability to perform physical examinations, difficulty demonstrating empathy and practicing or observing nonverbal communication are noteworthy drawbacks [14]. Moreover, teleconsultations have been shown to increase patient anxiety and discomfort, which could be further exacerbated by the presence of students [14,15]. Last but not least, patient confidentiality is also put at risk [1].

Universities, medical educators and healthcare providers must galvanize efforts to tackle these concerns. We propose the following suggestions. First, if telemedicine is to become a mainstay method of facilitating medical student exposure to cancer patients, medical schools should ensure sufficient student provisions and equal access to online resources. Second, undergraduate medical curricula should be revised to educate and train students on the use of telemedicine systems because students must be proficient in using these technologies as future physicians. Aside from technical training, students should also be educated on the limitations and uniqueness of using television-based systems. Communication skills, such as demonstrating empathy, must be adapted to teleconsultations. Students should be knowledgeable about video-call etiquette and how to set up an appropriate environment for themselves and patients [16]. Moreover, it is essential that upcoming changes in medical training are communicated to patients. If patients are aware of the reasons and the significance of including medical students in their care, they might be more willing to include them and be at ease during teleconsultations. Finally, regarding patient confidentiality, Elkaddoum et al. proposed the use of end-to-end encrypted software to prevent unwanted leaks [1]. Although unfamiliarity with this system may initially be an issue, providing dedicated training to students and medical personnel could easily overcome this.

Undergraduate medical oncology teaching in the UK has already been neglected enough amid the current crisis. Educators and physicians must realize the long-term consequences of marginalizing students during this pandemic. Medical students are the future generation of physicians, and thus they must be included in telemedicine promptly, even more so because these technologies are set to become a mainstay in the future. Medical schools must continue to adapt to the new face of medicine amid COVID-19, with larger emphasis placed on the transition to telemedicine education and training, particularly geared toward hospital exposure and clinical simulations [17].

\section{Author contributions}

AM Wozniak wrote the original draft and contributed to editing. KS Rallis was involved in conceptualization and editing of the letter.

Financial \& competing interests disclosure

The authors have no relevant affiliations or financial involvement with any organization or entity with a financial interest in or financial conflict with the subject matter or materials discussed in the manuscript. This includes employment, consultancies, honoraria, stock ownership or options, expert testimony, grants or patents received or pending, or royalties.

No writing assistance was utilized in the production of this manuscript.

\section{References}

1 Elkaddoum R, Haddad FG, Eid R, Kourie HR. Telemedicine for cancer patients during COVID-19 pandemic: between threats and opportunities. Future Oncol. 16(18), 1225-1227 (2020).

2 Wang C, Horby PW, Hayden FG, Gao GF. A novel coronavirus outbreak of global health concern. Lancet. 395(10223), 470-473 (2020). 
Davies NG, Klepac P, Liu Y et al. Age-dependent effects in the transmission and control of COVID-19 epidemics. Nat. med.26 1205-1211 (2020).

4 van de Haar J, Hoes LR, Coles CE et al. Caring for patients with cancer in the COVID-19 era. Nat. Med. 26(5), 665-671 (2020).

5 Hazin R, Qaddoumi I. Teleoncology: current and future applications for improving cancer care globally. Lancet Oncol. 11(2), 204-210 (2010).

6 Harky A, Chiu CM, Yau THL, Lai SHD. Cancer patient care during COVID-19. Cancer Cell. 37(6), 749-750 (2020).

7 O'Byrne L, Gavin B, McNicholas F. Medical students and COVID-19: the need for pandemic preparedness. J. Med. Ethics. 46(9), 623-626 (2020).

8 Mattes MD, Patel KR, Burt LM, Hirsch AE. A nationwide medical student assessment of oncology education. J. Cancer Ed. 31(4), 679-686 (2016).

9 Pavlidis N, Gatzemeier W, Popescu R et al. The masterclass of the European School of Oncology: the 'key educational event' of the school. Eur. J. Cancer. 46(12), 2159-2165 (2010).

10 Agarwal A, DeNunzio NJ, Ahuja D, Hirsch AE. Beyond the standard curriculum: a review of available opportunities for medical students to prepare for a career in radiation oncology. Int. J. Radiat. Oncol. Biol. Phys. 88(1), 39-44 (2014).

11 Erikson C, Salsberg E, Forte G, Bruinooge S, Goldstein M. Future supply and demand for oncologists: challenges to assuring access to oncology services. J. Oncol. Pract. 3(2), 79-86 (2007).

12 Kalia V, Srinivasan A, Wilkins L, Luker GD. Adapting scientific conferences to the realities imposed by COVID-19. Radiol. Imaging Cancer. 2(4), (2020).

13 Mayes J, Davies S, Harris A, Wray E, Dark GG. Impact of a 2-week oncology placement on medical students' perception of cancer. J. Cancer Ed. 33(1), 174-179 (2018).

14 Kitamura C, Zurawel-Balaura L, Wong RK. How effective is video consultation in clinical oncology? A systematic review. Curr. Oncol. 17(3), 17-27 (2010).

15 Hoek PD, Schers HJ, Bronkhorst EM, Vissers KCP, Hasselaar JGJ. The effect of weekly specialist palliative care teleconsultations in patients with advanced cancer - a randomized clinical trial. BMC Med. 15(1), 119 (2017).

16 Haney T, Kott K, Fowler C. Telehealth etiquette in home healthcare: the key to a successful visit. Home Healthc. Now. 33(5), 254-259 (2015).

17 Yaghobian S, Ohannessian R, Iampetro T et al. Knowledge, attitudes and practices of telemedicine education and training of French medical students and residents. J. Telemed Telecare. doi: 10.1177/1357633X20926829 (2020) (Epub aheadof print). 